OPEN ACCESS

Edited by:

Roger M. Bourne,

University of Sydney, Australia

Reviewed by:

Andre Bongers,

University of New South Wales,

Australia

Juan Antonio Hernandez-Tamames,

Erasmus University Rotterdam,

Netherlands

${ }^{*}$ Correspondence:

Ahmad Chaddad

ahmad.chaddad@mail.mcgill.ca

Tamim Niazi

tniazi@jgh.mcgill.ca

Specialty section:

This article was submitted to

Cancer Imaging and Diagnosis,

a section of the journal

Frontiers in Oncology

Received: 20 July 2018 Accepted: 04 December 2018

Published: 18 December 2018

Citation:

Chaddad A, Niazi T, Probst $S$, Bladou F, Anidjar M and Bahoric B (2018) Predicting Gleason Score of

Prostate Cancer Patients Using

Radiomic Analysis.

Front. Oncol. 8:630.

doi: $10.3389 /$ fonc. 2018.00630

\section{Predicting Gleason Score of Prostate Cancer Patients Using Radiomic Analysis}

\author{
Ahmad Chaddad ${ }^{1,2 *}$, Tamim Niazi $^{1 *}$, Stephan Probst ${ }^{3}$, Franck Bladou ${ }^{4}$, Maurice Anidjar ${ }^{4}$ \\ and Boris Bahoric ${ }^{1}$
}

${ }^{1}$ Division of Radiation Oncology, McGill University, Montreal, QC, Canada, ${ }^{2}$ Department of Automated Production Engineering, ETS, Montreal, QC, Canada, ${ }^{3}$ Division of Nuclear Medicine, McGill University, Montreal, QC, Canada,

${ }^{4}$ Depatment of Urology, McGill University, Montreal, QC, Canada

Purpose: Use of quantitative imaging features and encoding the intra-tumoral heterogeneity from multi-parametric magnetic resonance imaging (mpMRI) for the prediction of Gleason score is gaining attention as a non-invasive biomarker for prostate cancer (PCa). This study tested the hypothesis that radiomic features, extracted from mpMRI, could predict the Gleason score pattern of patients with PCa.

Methods: This analysis included T2-weighted (T2-WI) and apparent diffusion coefficient (ADC, computed from diffusion-weighted imaging) scans of $99 \mathrm{PCa}$ patients from The Cancer Imaging Archive (TCIA). A total of 41 radiomic features were calculated from a local tumor sub-volume (i.e., regions of interest) that is determined by a centroid coordinate of PCa volume, grouped based on their Gleason score patterns. Kruskal-Wallis and Spearman's rank correlation tests were used to identify features related to Gleason score groups. Random forest (RF) classifier model was used to predict Gleason score groups and identify the most important signature among the 41 radiomic features.

Results: Gleason score groups could be discriminated based on zone size percentage, large zone size emphasis and zone size non-uniformity values $(p<0.05)$. These features also showed a significant correlation between radiomic features and Gleason score groups with a correlation value of $-0.35,0.32,0.42$ for the large zone size emphasis, zone size non-uniformity and zone size percentage, respectively (corrected $p<0.05$ ). RF classifier model achieved an average of the area under the curves of the receiver operating characteristic (ROC) of 83.40, 72.71 , and $77.35 \%$ to predict Gleason score groups $(\mathrm{G} 1)=6 ; 6<(\mathrm{G} 2)<(3+4)$ and $(\mathrm{G} 3) \geq 4+3$, respectively.

Conclusion: Our results suggest that the radiomic features can be used as a non-invasive biomarker to predict the Gleason score of the PCa patients.

Keywords: biomarkers, classification, gleason score, radiomics, prostate cancer

\section{INTRODUCTION}

Prostate cancer $(\mathrm{PCa})$ is one of the most prevalent male malignancies in the developed countries and $1 / 6$ th of the men in the USA are expected to be diagnosed with this disease in their lifetime (1). Patients with localized PCa are classified into three risk groups (low, intermediate, and high risk) based on their prostate-specific Antigen (PSA) level, Gleason score and clinical stage 
(i.e., TNM) (2). For men with low-risk prostate cancer, active surveillance as opposed to immediate treatment has become a widely accepted treatment approach $(3,4)$. However, PCa has the capacity to progress over time. A study of 17,943 patients with low-risk PCa who were treated with radical prostatectomy (RP) revealed that upgrading and upstaging occurred in $45 \%$ of these men (5). Furthermore, the deferral of RP for more than 12 months has been associated with a 1.7-fold increased risk of non-organ confined disease after surgery (5).

Numerous studies have shown that PSA alone is not an accurate indicator of $\mathrm{PCa}$ and over-diagnosis is occurring in up to $50 \%$ of men $(6,7)$. The inaccuracy of PSA screening often leads to subsequent unnecessary biopsies that are not only costly but invasive with serious side effects, such as infection, erectile dysfunction and bleeding. Therefore, there is a pressing need for a non-invasive test to distinguish between PCa grades to improve delivery of high precision care for these patients.

MR imagery, especially multi-parametric sequences (i.e., T1WI, T2-WI, FLAIR ... etc.), have been widely used for diagnosis, staging and treatment monitoring of different tumor types (815). A newly structured Prostate Imaging Reporting and Data System (PI-RADS v2) showed that this scoring method could predict the risk of prostate cancer presence based on the MR images (16). However, PI-RADS v2 involves interpretation of the images based on the experience of the radiologists that carries inter-reader variability. Moreover, PI-RADS has been used to investigate the relation between imaging features and Gleason score (GS) $(9,10,17,18)$. The volume of PCa has been shown to be significantly different between GS $=6$ and GS $\geq 7 \mathrm{PCa}$ (17). In addition, two recent studies demonstrated the usefulness of the texture features based on gray level co-occurrence matrices (GLCMs) of MR images (i.e., T2WI) as an indicator for the pathological differences in PCa $(19,20)$. To date, there has been limited work investigating the link between the texture features of the PCa imaging and the GS.

Offering a non-invasive and low-cost automated technique for analysis of tumor properties based on MR images, radiomics has recently been used to interrogate the tumor heterogeneity of several types of cancer, such as $\operatorname{GBM}(21)$, lung $(22,23)$, colorectal $(24,25)$, and PCa $(17,26-28)$ among others. Radiomic features with machine learning models can analyze large numbers of PCa images that will overcome the limitations (i.e., inter-reader variability for interpretation of the ROIs within an image) for assessing and classifying PCa lesions. Specifically, many previous studies have used such machine learning models to assess for the PCa aggressiveness (13,29-32). Combined structural features and metabolic imaging data of PCa in mpMRI [i.e., T2-WI and magnetic resonance spectroscopy (MRS)] using the RF classifier, have shown the capacity of these models to detect the areas of cancer in a PCa tissue $(18,33)$. Extracted multitexture features derived from T1-WI, T2-WI, and DiffusionWeighted Imaging (DWI) to characterize the PCa tissue have been demonstrated to improve the classification rate of prostate tumor recognition (34-36). Differences between the cancer grades of a tumor (i.e., PCa) vs. non-tumor regions have been related to differences in heterogeneity (i.e., texture). For example, PCa can be discriminated from benign tissues and be detected based on a different histogram analysis, as shown by Vos et al (37). However, the dominant features that could assist in measuring the heterogeneity within PCa have not yet been fully studied.

We hypothesize that the comprehensive integration of radiomic features from mpMR images will identify new characteristics that are capable of distinguishing PCa with different GS groups whether in the transition or peripheral zones.

\section{MATERIALS AND METHODS}

The proposed pipeline of radiomic analysis to predict GS involves data acquisition from $\mathrm{T} 2-\mathrm{WI}$ and $\mathrm{ADC}$ images, automatic matching of $\mathrm{PCa}$ regions, radiomic features computation from determined subvolume of PCa tumor, and feature analysis. The proposed flowchart for radiomic analysis of PCa is shown in Figure 1. Kruskal-Wallis significance test and Spearman rank correlation were performed to identify radiomic features which were associated with GS groups. We then applied the RF classifier, using the radiomic features, to differentiate between the GS groups and rank the importance value of each radiomic features for prediction. The detailed methodology for each step of the proposed flowchart is described below.

\section{Patients and Data Acquisition}

We reviewed the $99 \mathrm{PCa}$ patients of the SPIE-AAPMNCI Prostate MR Gleason Grade Group Challenge (http:// spiechallenges.cloudapp.net/competitions/7) and the Cancer Imaging Archive (TCIA), a publicly available medical image repository. Note that the challenge consists of $162 \mathrm{PCa}(99$ training and 63 testing) patients. We considered only the 99 training cases where the Gleason score was available, previously de-identified by SPIE-AAPM-NCI, and the dataset is available for public download (i.e., Supplementary Table 1). The remaining 63 cases (i.e., testing) did not have Gleason score available. As such, no institutional review board or Health Insurance Portability and Accountability Act approval were required for our study. The dataset included T2-WI and ADC which were computed from DWI. The MR images were acquired on two different types of Siemens 3T MR scanners, the MAGNETOM Trio and Skyra. T2-weighted images were acquired using a turbo spin echo sequence and had a resolution of around $0.5 \mathrm{~mm}$ in the plane and a slice thickness of $3.6 \mathrm{~mm}$. The DWI series were acquired with a single-shot echo planar imaging sequence with a resolution of $2 \mathrm{~mm}$ in-plane and $3.6 \mathrm{~mm}$ slice thickness with diffusion-encoding gradients in three directions. Three b-values were acquired (50, 400, and 800 ), and the ADC map was subsequently calculated by the scanner software (https://wiki.cancerimagingarchive.net/). Gray-scale images were then intensity normalized to reduce the intensity variation between MRIs obtained from different acquisitions. Moreover, images were acquired at a resolution of $320 \times 320 \times 19$ voxels. Patient characteristics information is reported in Supplementary Table 2. The histograms of voxel intensity distribution across the T2-WI and ADC are not reliable for differentiating between the three Gleason Groups, (i.e., Supplementary Figure 1). The five groups of Gleason 


\section{Original mpMRI}

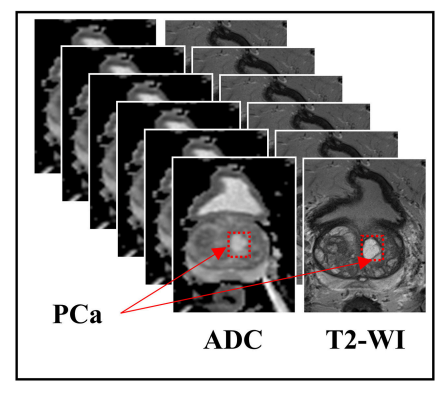

2. ROI

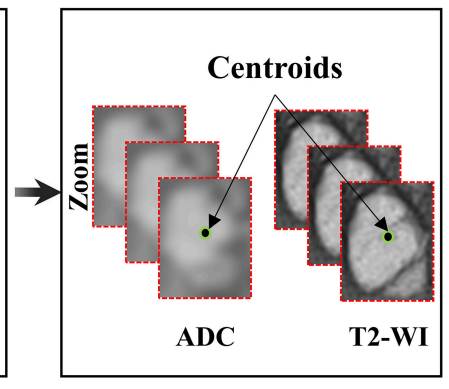

3. Feature extraction

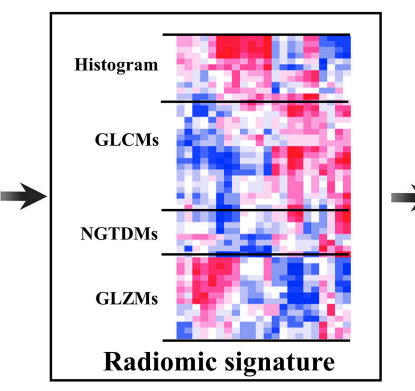

4. Statistical analysis

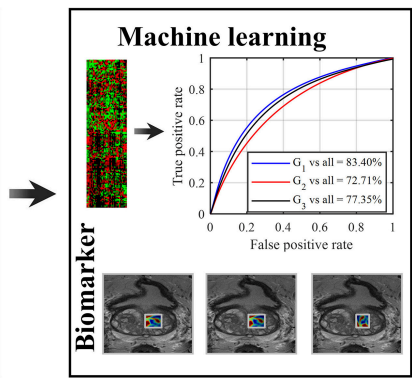

FIGURE 1 | Schema of a radiomic model for patients with PCa. Acquisition of pre-treatment PCa patient's MR images; Regions of interest (i.e., subvolume $21 \times 21 \times$ 3 voxels); Extraction of 41 radiomic features from ROls; Feature significance analysis based on Spearman rank correlation and Kruskal-Wallis, and multivariate prediction of Gleason score groups using the random forest model.

scores were divided into three groups-G1 when $\mathrm{GS} \leq 6$; G2 when $\mathrm{GS}=3+4$; and G3 when GS $\geq 4+3$. Therefore, we used several texture features derived from gray-level cooccurrence matrix (GLCM), neighborhood gray-tone difference matrix (NGTDM) and gray-level size zone matrix (GLSZM) that able to capture the subtle differences between the GS groups.

\section{Feature Extraction}

Regions of interest (ROI) of PCa were automatically selected based on the centroid coordinates of all the lesions in T2WI and ADC images that were provided by the SPIEAAPM-NCI Prostate MR Gleason Grade Group Challenge. For each patient, a sub-volume (i.e., ROI) of $21 \times 21 \times$ 3 voxels was collected separately, from the axial image in T2-WI and ADC to ensure accuracy and precision. Each sub-volume is encoded into a set of features by applying the 41 radiomic features as following: six intensity features derived from histogram and 35 texture features derived from GLCMs (38, 39), NGTDM, (40), GLSZM (41) as shown in Supplementary Table 3.

These features measure various textural properties and quantify the hidden patterns in the ROI. To capture more meaningful patterns of texture, image intensities of ROIs were uniformly quantized to 32 gray levels prior to computing the features. These features are described in several previous studies $(22,38-41)$ and detailed description of each feature is listed in their Supplementary Materials $(22,42)$. Features were extracted separately from T2-WI and ADC images and the average of each feature across the two images (i.e., T2-WI and ADC) was considered.

\section{Statistical Analysis}

To identify the significant radiomic features in comparing between the three groups of GS (i.e., G1, G2, G3), we first applied the Kruskal-Wallis test on each radiomic feature before multiple corrections using the Holm-Bonferroni method (43) to identify significant features $(P<0.05)$. We then used Spearman's rank correlation (44) to compute the correlation value $(\rho)$ between each of radiomic features and the GS group for the corresponding PCa patients. The rank correlation value was obtained between the GS group and each radiomic feature, with values between \pm 0.3 and \pm 0.5 indicating moderate (i.e., middle) correlation. We measured the significance of these correlation values based on the null hypothesis that there is no correlation. As in the previous test, we corrected $p$-values using the Holm-Bonferroni procedure and considered correlation significant if they reached a $p<0.05$ after correction.

We considered all 41 radiomic features as the input for the RF classifier model (45) and performed multivariate analysis for classifying the patients into three groups of GS [G1 vs. all (G2G3); G2 vs. all (G1-G3); G3 vs. all (G1-G2)]. We utilized RF for our analysis since it is one of the most effective classification models and leads to a low bias/variance classification result. In addition, the RF model in training algorithm possesses a feature selection process that allows assessment of each input feature (46). We acknowledge however that various other classifier models could be used for this task.

To report unbiased metrics, we used a 5-fold cross-validation strategy, where training features are divided into 5 equal sized subsets and, in each validation run, one subset is put aside for testing and the remaining 4 subsets are used to train the RF classifier. Each of these subsets was used in turn to compute the performance metrics of the trained RF model of the remaining samples using the 500 decision trees [i.e., number of trees within the RF model (47)]. Performance metrics [i.e., area under the curve (AUC), classifier accuracy, negative predictive value (NPV) and positive predictive value (PPV)], are then reported as the average obtained across 5-folds.

To compute the importance of each of the 41 features in individual classification group [G1 vs. all (G2-G3); G2 vs. all (G1-G3); G3 vs. all (G1-G2)], we measured the increase in prediction error resulting from the permutation of feature values across out-of-bag observations. The importance values were computed for every $\mathrm{RF}$ tree and averaged over the entire ensemble. These values were then normalized by dividing them by the ensemble's standard deviation. Finally, the importance of each of the 41 features was obtained by 
averaging these normalized values across all 5-folds. Positive importance value indicates that the feature is predictive, whereas negative importance value identifies features with no predictive value.

\section{RESULTS}

\section{Patients Characteristics and Data Acquisition}

In this study we used a retrospective dataset comprising of $99 \mathrm{PCa}$ patients collected from the SPIE-AAPM-NCI and the Cancer Imaging Archive (TCIA). Each patient's tumor lesion has a pathology Gleason Grade Group (GGG) number, which consists of five groups defined previously by Esptein JI et al. (48). However, we reclassified the patients based on their GGG into three groups to better represent clinical management: Group 1 (G1), 30 patients, Gleason score 6; Group 2 (G2), 39 patients, Gleason score $3+4$; and Group 3 (G3), 30 patients, Gleason primary pattern of 4 or higher $(4+3,8$, 9 or 10). The relevant patient characteristics are reported in Supplementary Table 2.

\section{Radiomic Features and Association With Gleason Score}

After extracting 41 radiomic features from MR images of each PCa patient, we applied univariate analysis using the Kruskal-Wallis significance test to determine if any individual radiomic feature was statistically significant to compare between the GS groups. We also computed the Spearman's rank correlation coefficient between the radiomic features and GS groups.

In Kruskal-Wallis significance test, we found only three features, namely, large zone size emphasis, zone size nonuniformity and zone size percentage, that were statistically significant difference between the three GS groups (G1, G2 and G3): $p<0.05$. None of the remaining features were statistically significant to compare between GS groups following Holm-Bonferroni correction (Figure 2A; Table 1). Spearman's rank correlation, applied between radiomic features and GS groups showed the significant moderate correlation values $(\rho)$ of $-0.35,0.32$, and 0.42 for the large zone size emphasis, zone size non-uniformity and zone size percentage, respectively with a corrected $p<0.05$. The correlation values of remaining radiomic features were not statistically significant following Holm-Bonferroni correction (Figure 2B; Table 2). The significantly correlated features were similar to those which could distinguish between GS groups.

In order to show the changes in the original five Gleason score groups, we repeated the Kruskal-Wallis significance test and the Spearman correlation. Results can be found in Supplementary Figure 2, which shows the $p$-values in $\log _{10}$ scale and the correlation value. In general, two radiomic features were statistically significant to be associated with five GS groups [large zone size emphasis $(\rho=-0.35)$ and zone size percentage ( $\rho=$ $0.43)$ ] which are similar to those obtained using the three GS groups.

\section{Classification of GS Groups}

Using all the radiomic features (i.e., 41 features) as input for the RF classifier model to predict the GS groups of the $99 \mathrm{PCa}$ patients, the classifier accuracy was $81.82 \%$ (75.00-84.00\%) for G1 patients, $66.67 \%$ (57.89-72.13\%) for G2 patients and $74.75 \%$ (80.56-59.26\%) for G3 patients (Supplementary Table 4). There was a higher value of the AUC of $83.40 \%$ to predict G1 compared to $72.71 \%$ and $77.35 \%$ when using radiomic features of G2 and G3, respectively (Figure 3A). The confusion matrix which reveals the RF classifier misclassification rate is shown in Supplementary Table 5. Correct classification of GS groups

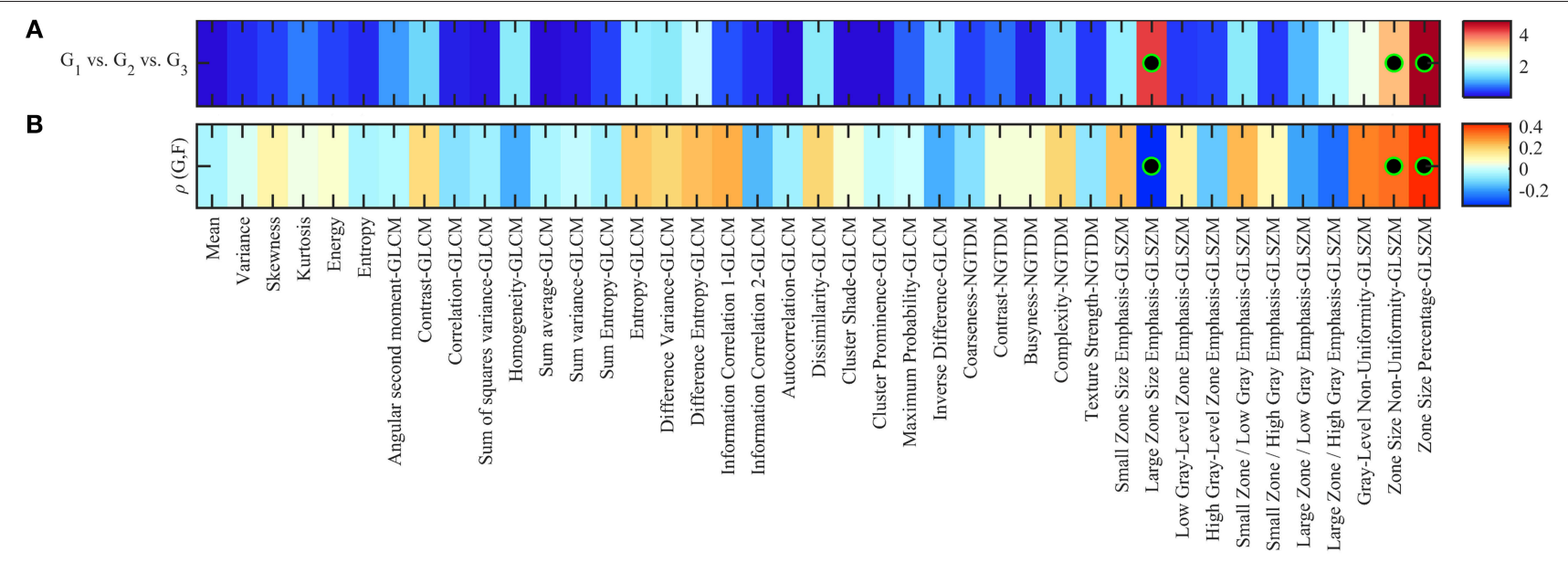

FIGURE 2 | (A) Heatmap of Kruskal-Wallis significance test $p$-values (-log 10 scale) using radiomic features to identify patients of different Gleason scores. Significant features to compare between Gleason score groups are indicated with a black-green circle (corrected $p<0.05$ ). (B) Spearman rank correlation between feature value and groups of Gleason score (i.e., 1, 2, 3), color-coded from minimum (dark blue) to maximum (dark red). Features with statistically significant correlation (i.e., corrected $p<0.05$ ) are indicated with a black-green circle. 
TABLE 1 | Comparisons of radiomic features related to the Gleason score groups of prostate cancer.

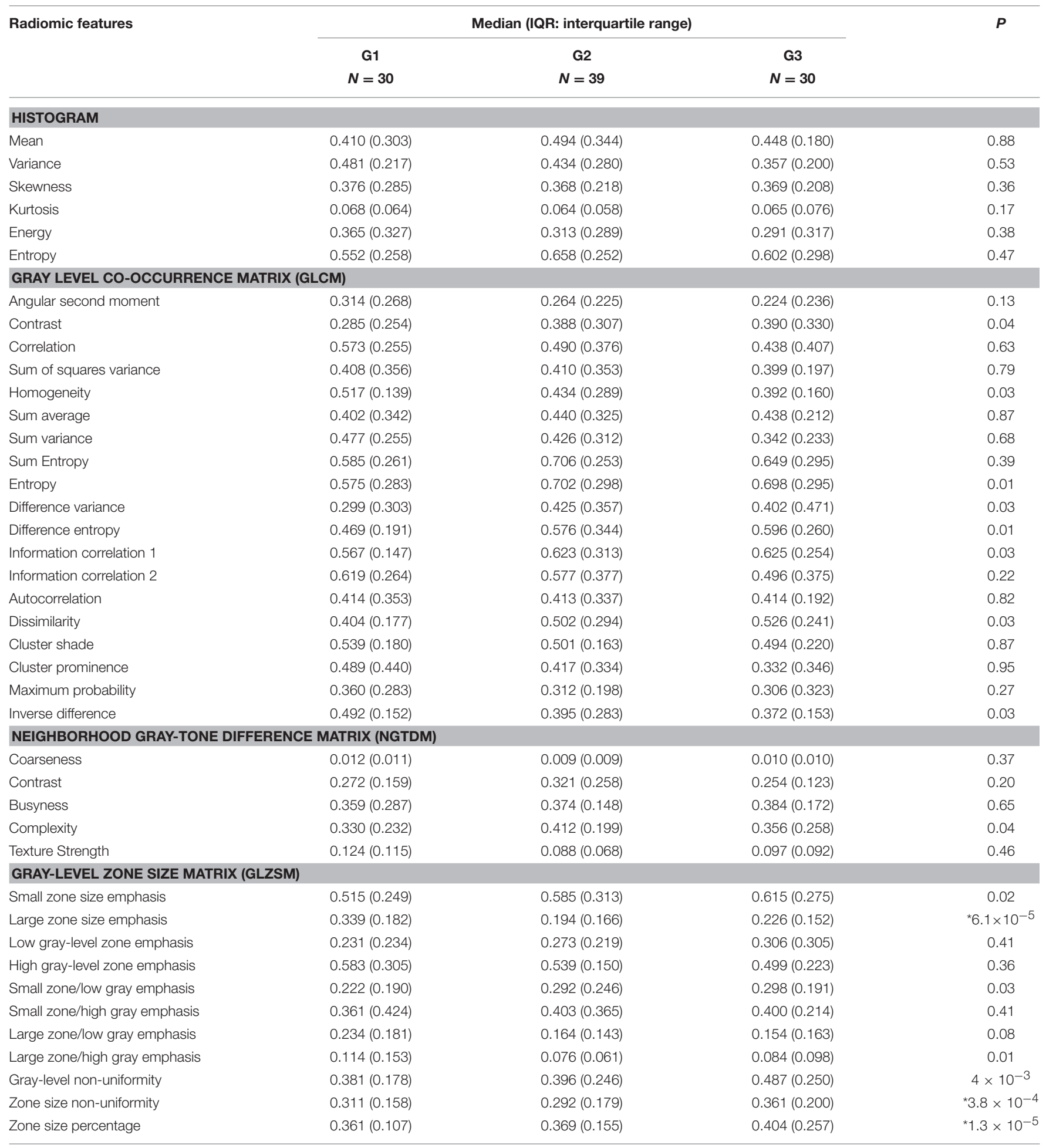

*Significant features following Holm-Bonferroni correction; G1, G2, and G3 is the Gleason score of the group 1, 2, and 3, respectively.

was achieved for 81/99 (18 G1 and 63 G2-G3), 66/99 (22 G2 and 44 G1-G3) and 74/99 (16 G3 and 58 G1-G2) of patients.

To validate our predictive model, we randomly assigned the 99 PCa patients to two datasets groups of balanced classes that were used as training $(n=40)$ and test $(n=20)$ datasets. Using the trained RF model to test the new datasets $(n=20)$, a higher value of the AUC of $89.73 \%$ to predict G1 compared to 66.64 and $63.94 \%$ when using radiomic features of G2 and G3, respectively (Figure 3B). 


\section{Importance Features for Each of GS Groups}

Features based on the RF classifier model for classifying patients into GS groups are depicted in Figure 4. We found that 40/41 radiomic features have an importance value $>0$, green bars, to classify G1 from G2 and G3 patients (Figure 4A). The most important features contributing to G1 identification were zone size percentage, large zone size emphasis, and zone size nonuniformity.

To distinguish G2 from G1 and G3, Figure 4B, 21 radiomic features had an importance value $>0$. We noticed that the entropy was the most dominant feature for predicting the G2 group. While 20 features had a negative importance value $<0$, red bars. To distinguish G3 from G2 and G1, 27 features had an importance value $>0$ and the sum-entropy with energy-histogram were the most important features, while 14 features had a negative importance value, Figure 4C. The entropy and sum entropy features were the most important features to predict the G2 and G3, respectively. These features

TABLE 2 | Correlated features with Gleason score groups.

\begin{tabular}{lc}
\hline Features & $\boldsymbol{\rho}(\boldsymbol{p})$ \\
\hline Large zone size emphasis-GLSZM & $-0.35\left(3.1 \times 10^{-4^{*}}\right)$ \\
Zone size non-uniformity & $0.32\left(9.01 \times 10^{-4^{*}}\right)$ \\
Zone size percentage-GLSZM & $0.42\left(1.1 \times 10^{-5^{*}}\right)$ \\
\hline
\end{tabular}

*Significant features following Holm-Bonferroni correction; $\rho$ is the correlation coefficient. (i.e., entropy and sum entropy) describe tissue heterogeneity and measure the randomness of texture within the PCa region.

\section{Features Analysis for Predicting GS}

To analyse the impact of the features in predicting the GS of patients, we repeated the classification (training/testing $=40 / 20$ ) between GS groups using the features derived from ADC and T2-WI images. We found that the AUC values of GS $<6$ and GS $\geq 7$ using the features derived from T2-WI images are higher with 84.12 and $63.91 \%$ to those derived from ADC with 69.95 and $58.41 \%$, respectively. While the AUC value of GS $=7$ (or $3+4)$ using the features derived from ADC images is higher with $62.09 \%$ comparing to $54.92 \%$ that derived from T2-WI images (Figures 5A,B). In general, the most important features for predicting the GS are derived from T2-WI images (Figure 5C).

\section{DISCUSSION}

Clinicians are trained for the ability to diagnose malignant disease through the visual study of MRI scans. However, visual methods are subjective, prone to errors and low throughput, a challenge which is becoming more of a limitation as the burden on healthcare resources expand with the aging population. Radiomic analysis, involving feature extraction from many images with classifier techniques, can automatically predict the grade of cancer with a precision and speed beyond the scope of human visual analysis. Several studies have used
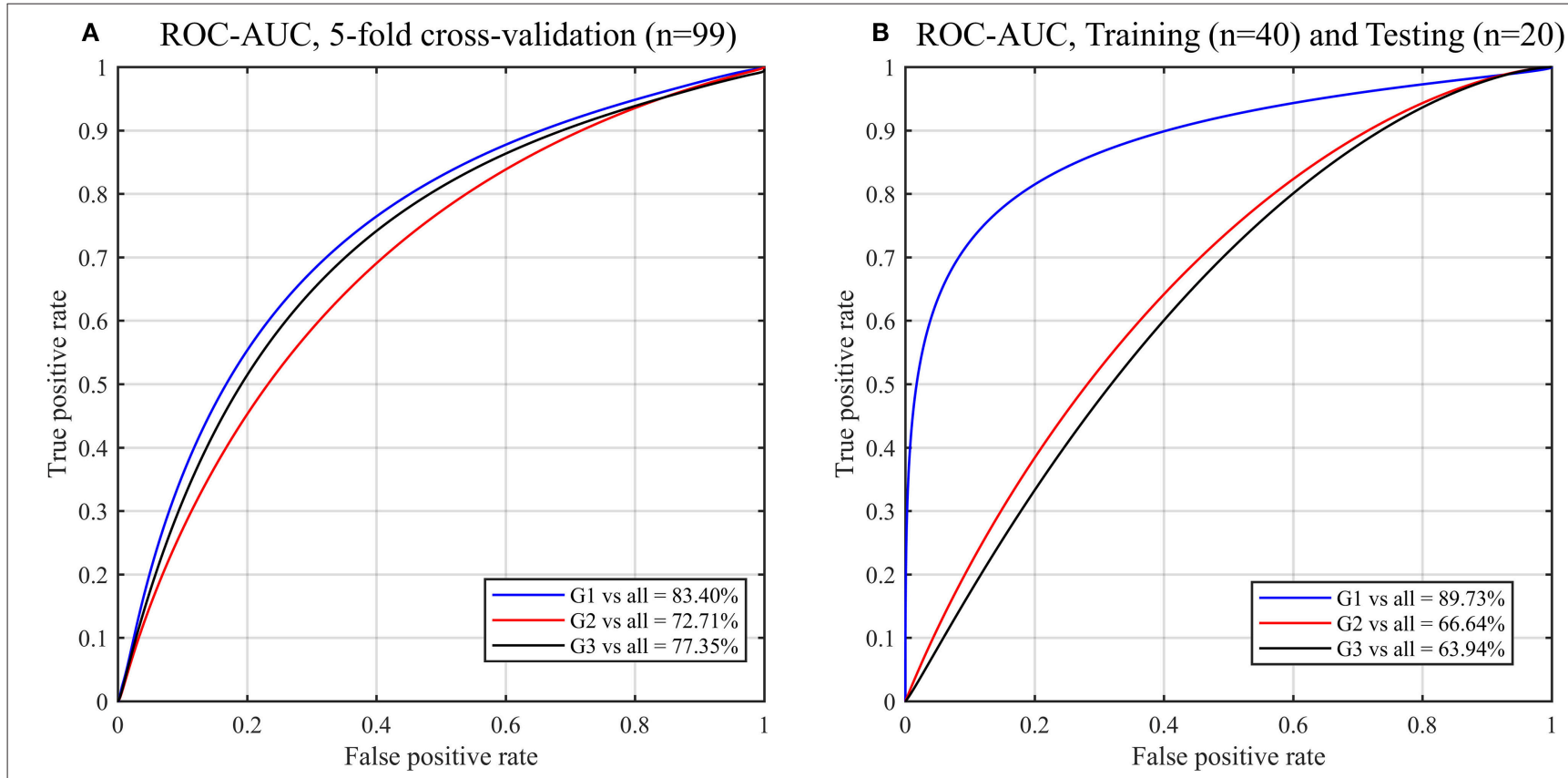

FIGURE 3 | ROC curves and AUC obtained by the random forest (RF) models for predicting Gleason score of PCa patients using the radiomic features. Gleason score groups: G1 (group 1), G2 (group 2), G3 (group 3). (A) 5-fold cross validation, (B) Validation of the trained ( $n=40$; balanced classes) RF model by testing new datasets $(n=20)$. 


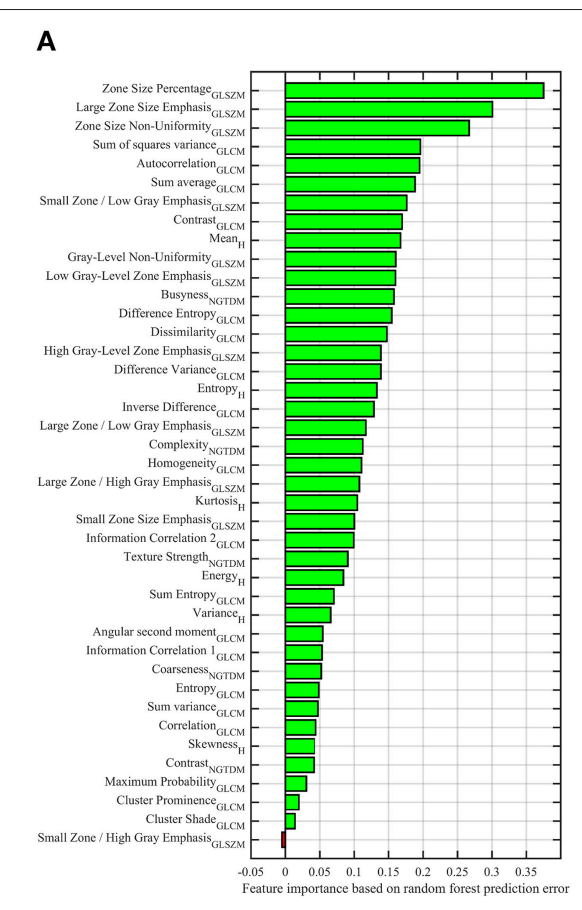

B

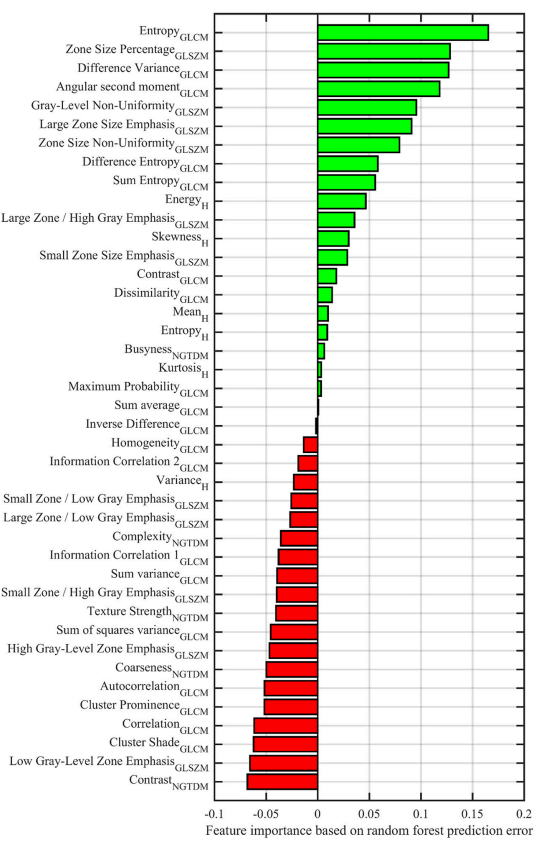

C

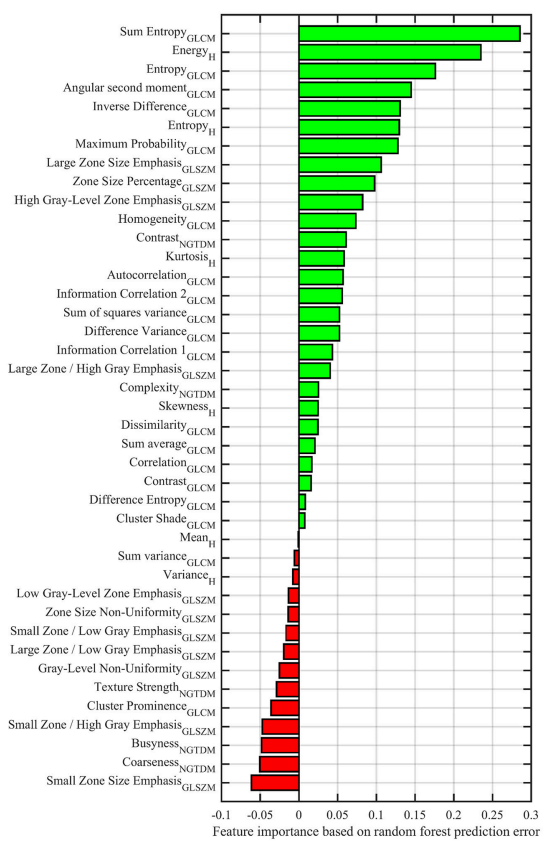

FIGURE 4 | Importance of features for predicting the Gleason score of PCa patients, G1 vs. G2-G3 (A), G2 vs. G1-G3 (B), G3 vs. G1-G2 (C). Reported values correspond to the average increase in prediction error obtained by permuting the values of individual features across out-of-bag observations (46). Green and red bars represent the positive and negative impact for predicting the Gleason score groups.

radiomic features, derived from $\mathrm{MR}$ images, for computerassisted diagnosis $(29,31,32,49,50)$. In addition to providing basic diagnostic information, such analysis may also reveal insights into the underlying heterogeneity of cancers, making further investigation into the radiomic assessment of PCa a priority. Radiomics has the additional benefit of automation, which can reduce human effort and cost whilst preventing patient morbidity and mortality associated with misdiagnosis and under/over treatment. However, radiomic features most helpful in predicting GS of PCa to estimate the aggressivity of a tumor remain largely unexplored.

In this study, we used three different methods: (i) KruskalWallis significance test, (ii) Spearman rank correlation coefficient, and (iii) RF classifier model to test whether radiomics can successfully identify GS of PCa patients. Comparison of radiomic features between the three groups of GS revealed three radiomic features (i.e., large zone size emphasis, zone size non-uniformity and zone size percentage) with the capacity to discriminate between GS groups with a corrected $p<$ 0.05. The similar three features were moderately correlated with GS groups with a corrected $p<0.05$. Our findings confirm that the three features were associated with GS groups of PCa.

Binary classification using RF model demonstrated that similar features which were previously shown to be significantly correlated with GS groups are the most important (i.e., dominant) features for the prediction of patients with a GS $=6$. These three features describe the homogeneity of the images through the size of uniform voxel regions in different PCa lesions. However, entropy and sum entropy features were demonstrated to have the greatest importance for predicting $\mathrm{G} 2(\mathrm{GS}=[3+4])$ and $\mathrm{G} 3(\mathrm{GS}=[4+3,8,9,10])$ of PCa patients, respectively. Specifically, entropy and sum entropy features describe the randomness of the texture or the abnormalities of the PCa regions. The highest values of entropy features in G2 and G3 are linked to the abnormality in texture (i.e., heterogeneity) that is related to tumor (i.e., PCa) aggressiveness.

Our findings are consistent with several previous studies that utilized texture analysis. Haralick's texture features were demonstrated to be useful for PCa detection and GS assessment. Specifically, GS was associated with higher entropy features (27). Combined analysis of T2-WI images and MRS images demonstrated the feasibility for radiomics to discriminate between benign vs. cancerous and high vs. low GS using 29 preoperative mpMRI (i.e., T2-WI and MRS) (18). We observed that several previous studies have focused on the classification between the structure of benign and cancerous regions (51). This is consistent with our study in considering the Gleason score as the baseline indicator for classifying non-cancerous prostate from malignant cancers.

Our study has several limitations. Our analysis was performed on a retrospective analysis of a small group of patients ( $n=99$ ), including ADC and T2-WI MRI images only. More image modalities, such as proton density-weighted (PDW) and dynamic contrast enhanced (DCE) could potentially 


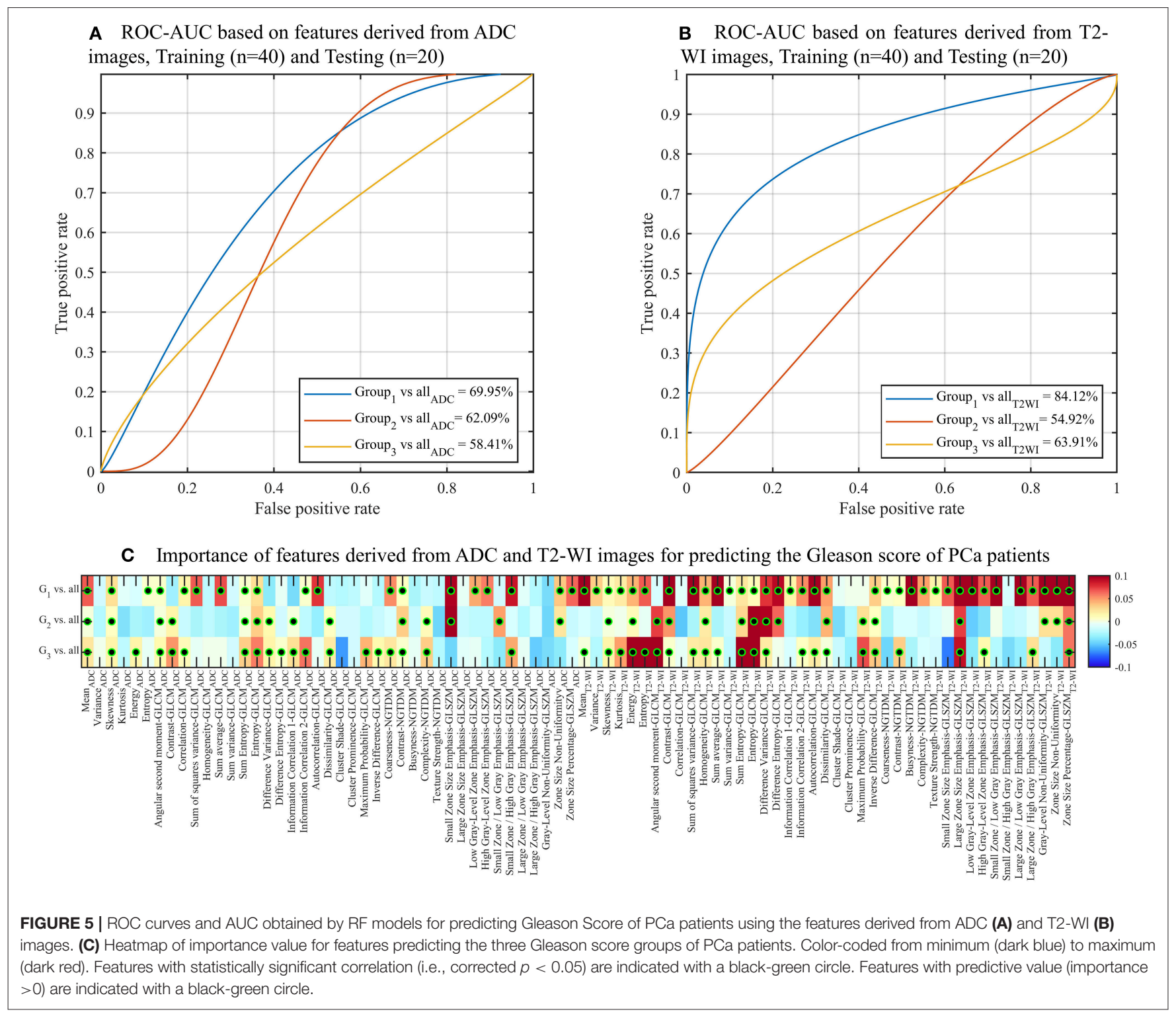

improve the performance metrics for predicting the GS. Then, the results require external validation on a larger scale prior to broader clinical application. We considered only 41 different image-based features including first- and second-order textures that derived from sub-volume ROI without considering the manual segmentation to eliminate any bias resulting from inter-reader variability. PIRADS is an extensively studied and validated system, and any potential replacement needs to be compared to it as the current imaging standard.

Future work could explore the shape features (e.g., volume) of the full PCa area. Machine learning techniques, such as deep radiomics (52) based on convolutional neural networks could be also employed to learn discriminative features in a more data-driven manner.

\section{CONCLUSIONS}

In this study, we presented the radiomic features that were computed from both ADC and T2-WI images for the discrimination between three groups of GS and classifying these groups using the RF classifier model. Our results suggest that only three features (i.e., zone size percentage, large zone size emphasis, and zone size non-uniformity) are able to identify groups of GS and significantly correlate each group. These three features appeared to be the most important to predict GS $\leq 6$, while the sum entropy was the most important feature to predict a GS $\geq 7$ $(4+3)$. Radiomic analysis has the potential to be used as a noninvasive test to predict GS for patients with PCa and therefore, further prospective studies are warranted to validate and confirm our findings. 


\section{AUTHOR CONTRIBUTIONS}

AC performed the experiments, analyzed data and wrote the paper. All authors reviewed the paper and gave final approval of the manuscript.

\section{REFERENCES}

1. Bard RL, Fütterer JJ, Sperling D. Image Guided Prostate Cancer Treatments. Berlin; Heidelberg: Springer (2014).

2. D’Amico AV, Moul J, Carroll PR, Sun L, Lubeck D, Chen MH. Cancer-specific mortality after surgery or radiation for patients with clinically localized prostate cancer managed during the prostate-specific antigen era. J Clin Oncol. (2003) 21:2163-72. doi: 10.1200/JCO.2003.01.075

3. Klotz L, Zhang L, Lam A, Nam R, Mamedov A, Loblaw A. Clinical results of long-term follow-up of a large, active surveillance cohort with localized prostate cancer. J Clin Oncol. (2010) 28:126-31. doi: 10.1200/JCO.2009.24.2180

4. Klotz L, Vesprini D, Sethukavalan P, Jethava V, Zhang L, Jain S, et al. Longterm follow-up of a large active surveillance cohort of patients with prostate cancer. J Clin Oncol. (2015) 33:272-7. doi: 10.1200/JCO.2014.55.1192

5. Weiner AB, Patel SG, Eggener SE. Pathologic outcomes for low-risk prostate cancer after delayed radical prostatectomy in the United States. Urol Oncol. (2015) 33:164.e11-17. doi: 10.1016/j.urolonc.2014.12.012

6. Andriole GL, Crawford ED, Grubb RLI, Buys SS, Chia D, Church TR, et al. Mortality results from a randomized prostate-cancer screening trial. $N$ Engl J Med. (2009) 360:1310-9. doi: 10.1056/NEJMoa0810696

7. Schröder FH, Hugosson J, Roobol MJ, Tammela TLJ, Ciatto S, Nelen V, et al. Screening and prostate-cancer mortality in a randomized European study. $N$ Engl J Med. (2009) 360:1320-8. doi: 10.1056/NEJMoa0810084

8. Soylu FN, Peng Y, Jiang Y, Wang S, Schmid-Tannwald C, Sethi I, et al. Seminal vesicle invasion in prostate cancer: evaluation by using multiparametric endorectal MR imaging. Radiology (2013) 267:797-806. doi: 10.1148/radiol.13121319

9. Wang Q, Li H, Yan X, Wu CJ, Liu XS, Shi HB, et al. Histogram analysis of diffusion kurtosis magnetic resonance imaging in differentiation of pathologic Gleason grade of prostate cancer. Urol Oncol. (2015) 33:337.e1524. doi: 10.1016/j.urolonc.2015.05.005

10. Hambrock T, Somford DM, Huisman HJ, van Oort IM, Witjes JA, Hulsbergenvan de Kaa CA, et al. Relationship between apparent diffusion coefficients at 3.0-T MR imaging and Gleason grade in peripheral zone prostate cancer. Radiology (2011) 259:453-61. doi: 10.1148/radiol.11091409

11. Wang J, Wu CJ, Bao ML, Zhang J, Wang XN, Zhang YD. Machine learning-based analysis of MR radiomics can help to improve the diagnostic performance of PI-RADS v2 in clinically relevant prostate cancer. Eur Radiol. (2017) 27:4082-90. doi: 10.1007/s00330-017-4800-5

12. Chung AG, Khalvati F, Shafiee MJ, Haider MA, Wong A. Prostate cancer detection via a quantitative radiomics-driven conditional random field framework. IEEE Access. (2015) 3:2531-41. doi: 10.1109/ACCESS.2015.2502220

13. Ginsburg SB, Algohary A, Pahwa S, Gulani V, Ponsky L, Aronen HJ, et al. Radiomic features for prostate cancer detection on MRI differ between the transition and peripheral zones: preliminary findings from a multi-institutional study. J Magn Reson Imaging (2017) 46:184-93. doi: 10.1002/jmri.25562

14. Lin YC, Lin G, Hong JH, Lin YP, Chen FH, Ng SH, et al. Diffusion radiomics analysis of intratumoral heterogeneity in a murine prostate cancer model following radiotherapy: pixelwise correlation with histology. J Magn Reson Imaging (2017) 46:483-9. doi: 10.1002/jmri.25583

15. Park SY, Kim CK, Park BK, Park W, Park HC, Han DH, et al. Early changes in apparent diffusion coefficient from diffusion-weighted MR imaging during radiotherapy for prostate cancer. Int J Radiat Oncol Biol Phys. (2012) 83:74955. doi: 10.1016/j.ijrobp.2011.06.2009

16. Park SY, Oh YT, Jung DC, Cho NH, Choi YD, Rha KH, et al. Prediction of biochemical recurrence after radical prostatectomy with PI-RADS

\section{SUPPLEMENTARY MATERIAL}

The Supplementary Material for this article can be found online at: https://www.frontiersin.org/articles/10.3389/fonc. 2018.00630/full\#supplementary-material

version 2 in prostate cancers: initial results. Eur Radiol. (2016) 26:2502-9. doi: 10.1007/s00330-015-4077-5

17. Donati OF, Afaq A, Vargas HA, Mazaheri Y, Zheng J, Moskowitz CS, et al. Prostate MRI: evaluating tumor volume and apparent diffusion coefficient as surrogate biomarkers for predicting tumor Gleason score. Clin Cancer Res. (2014) 20:3705-11. doi: 10.1158/1078-0432.CCR-14-0044

18. Tiwari P, Kurhanewicz J, Madabhushi A. Multi-kernel graph embedding for detection, Gleason grading of prostate cancer via MRI/MRS. Med Image Anal. (2013) 17:219-35. doi: 10.1016/j.media.2012.10.004

19. Nketiah G, Elschot M, Kim E, Teruel JR, Scheenen TW, Bathen TF, et al. T2-weighted MRI-derived textural features reflect prostate cancer aggressiveness: preliminary results. Eur Radiol. (2017) 27:3050-9. doi: 10.1007/s00330-016-4663-1

20. Fehr D, Veeraraghavan H, Wibmer A, Gondo T, Matsumoto K, Vargas $\mathrm{HA}$, et al. Automatic classification of prostate cancer Gleason scores from multiparametric magnetic resonance images. Proc Natl Acad Sci USA. (2015) 112:E6265-73. doi: 10.1073/pnas.1505935112

21. Chaddad A, Daniel P, Desrosiers C, Toews M, Abdulkarim B. Novel radiomic features based on joint intensity matrices for predicting glioblastoma patient survival time. IEEE J Biomed Health Inform. (2018). doi: 10.1109/JBHI.2018.2825027. [Epub ahead of print].

22. Chaddad A, Desrosiers C, Toews M, Abdulkarim B, Chaddad A, Desrosiers C, et al. Predicting survival time of lung cancer patients using radiomic analysis. Oncotarget (2017) 8:104393-407. doi: 10.18632/oncotarget.22251

23. Aerts HJWL, Velazquez ER, Leijenaar RTH, Parmar C, Grossmann P, Carvalho $\mathrm{S}$, et al. Decoding tumour phenotype by noninvasive imaging using a quantitative radiomics approach. Nature Comm. (2014) 5:4006. doi: 10.1038/ncomms5006

24. Chaddad A, Daniel P, Niazi T. Radiomics evaluation of histological heterogeneity using multiscale textures derived from 3D wavelet transformation of multispectral images. Front Oncol. (2018) 8:96. doi: 10.3389/fonc.2018.00096

25. Chaddad A, Desrosiers C, Bouridane A, Toews M, Hassan L, Tanougast C. Multi texture analysis of colorectal cancer continuum using multispectral imagery. PLOS ONE (2016) 11:e0149893. doi: 10.1371/journal.pone.0149893

26. Vargas HA, Akin O, Franiel T, Mazaheri Y, Zheng J, Moskowitz C, et al. Diffusion-weighted endorectal MR imaging at $3 \mathrm{~T}$ for prostate cancer: tumor detection and assessment of aggressiveness. Radiology (2011) 259:775-84. doi: 10.1148/radiol.11102066

27. Wibmer A, Hricak H, Gondo T, Matsumoto K, Veeraraghavan H, Fehr D, et al. Haralick texture analysis of prostate MRI: utility for differentiating non-cancerous prostate from prostate cancer and differentiating prostate cancers with different Gleason scores. Eur Radiol. (2015) 25:2840-50. doi: 10.1007/s00330-015-3701-8

28. Chaddad A, Kucharczyk MJ, Niazi T. Multimodal radiomic features for the predicting gleason score of prostate cancer. Cancers (2018) 10:E249. doi: 10.3390/cancers10080249

29. Peng Y, Jiang Y, Yang C, Brown JB, Antic T, Sethi I, et al. Quantitative analysis of multiparametric prostate MR images: differentiation between prostate cancer and normal tissue and correlation with Gleason score-a computer-aided diagnosis development study. Radiology (2013) 267:787-96. doi: 10.1148/radiol.13121454

30. Zhang YD, Wang J, Wu CJ, Bao ML, Li H, Wang XN, et al. An imagingbased approach predicts clinical outcomes in prostate cancer through a novel support vector machine classification. Oncotarget (2016) 7:78140-51. doi: 10.18632/oncotarget.11293

31. Stoyanova R, Takhar M, Tschudi Y, Ford JC, Solórzano G, Erho N, et al. Prostate cancer radiomics and the promise of radiogenomics. Transl Cancer Res. (2016) 5:432-447. doi: 10.21037/tcr.2016.06.20 
32. Scheltema MJ, Chang JI, van den Bos W, Böhm M, Delprado W, Gielchinsky I, et al. Preliminary diagnostic accuracy of multiparametric magnetic resonance imaging to detect residual prostate cancer following focal therapy with irreversible electroporation. Eur Urol Focus (2017). doi: 10.1016/j.euf.2017.10.007. [Epub ahead of print].

33. Madabhushi A, Feldman MD, Metaxas DN, Tomaszeweski J, Chute D. Automated detection of prostatic adenocarcinoma from highresolution ex vivo MRI. IEEE Trans Med Imaging (2005) 24:1611-25. doi: 10.1109/TMI.2005.859208

34. Duda D, Kretowski M, Mathieu R, Crevoisier R de, Bezy-Wendling J. MultiImage Texture Analysis in Classification of Prostatic Tissues from MRI. Preliminary Results. In: Pietka E, Kawa J, Wieclawek W, editors. Information Technologies in Biomedicine, Volume 3 Advances in Intelligent Systems and Computing. Cham: Springer (2014). p. 139-150.

35. Litjens G, Debats O, Barentsz J, Karssemeijer N, Huisman H. Computeraided detection of prostate cancer in MRI. IEEE Trans Med Imaging (2014) 33:1083-92. doi: 10.1109/TMI.2014.2303821

36. Khalvati F, Wong A, Haider MA. Automated prostate cancer detection via comprehensive multi-parametric magnetic resonance imaging texture feature models. BMC Med Imaging (2015) 15: doi: 10.1186/s12880-015-0069-9

37. Vos PC, Barentsz JO, Karssemeijer N, Huisman HJ. Automatic computer-aided detection of prostate cancer based on multiparametric magnetic resonance image analysis. Phys Med Biol. (2012) 57:1527-42. doi: 10.1088/0031-9155/57/6/1527

38. Haralick RM. Statistical and structural approaches to texture. Proc IEEE (1979) 67:786-804. doi: 10.1109/PROC.1979.11328

39. Gao X, Qian Y, Hui R, Loomes M, Comley R, Barn B, et al. Texturebased $3 \mathrm{D}$ image retrieval for medical applications. in IADIS International Conference e-Health. (2010). 101-108. Available online at: http://image.mdx. ac.uk/mirage2011/ehealth_2010.pdf (Accessed October 16, 2016)

40. Amadasun M, King R. Textural features corresponding to textural properties. IEEE Trans Syst Man Cybernet. (1989) 19:1264-74. doi: 10.1109/21.44046

41. Thibault G, Fertil B, Navarro CL, Pereira S, Cau P, Lévy N, et al. Texture indexes and gray level size zone matrix. Application to cell nuclei classification. In: 10th International Conference on Pattern Recognition and Information Processing, PRIP 2009 (Minsk, Belarus) (2009). p. 140-145. Available at: https://hal.archives-ouvertes.fr/hal-01499715 (Accessed April 3, 2018).

42. Chaddad A, Desrosiers C, Hassan L, Tanougast C. Hippocampus and amygdala radiomic biomarkers for the study of autism spectrum disorder. BMC Neurosci. (2017) 18:52. doi: 10.1186/s12868-017-0373-0

43. Holm S. A simple sequentially rejective multiple test procedure. Scandinavian J Statist. (1979) 6:65-70. doi: 10.2307/4615733
44. Zar JH. Significance testing of the spearman rank correlation coefficient. J Am Statist Assoc. (1972) 67:578-80. doi: 10.1080/01621459.1972.10481251

45. Breiman L. Random forests. Machine Learn. (2001) 45:5-32. doi: 10.1023/A:1010933404324

46. Archer KJ, Kimes RV. Empirical characterization of random forest variable importance measures. Computat Statist Data Analy. (2008) 52:2249-60. doi: 10.1016/j.csda.2007.08.015

47. Oshiro TM, Perez PS, Baranauskas JA. How many trees in a random forest? In: Machine Learning and Data Mining in Pattern Recognition Lecture Notes in Computer Science. Berlin; Heidelberg: Springer (2012). p. 154-168.

48. Epstein JI, Egevad L, Amin MB, Delahunt B, Srigley JR, Humphrey PA, Grading Committee. The 2014 International Society of Urological Pathology (ISUP) consensus conference on gleason grading of prostatic carcinoma: definition of grading patterns and proposal for a new grading system. Am J Surg Pathol. (2016) 40:244-52. doi: 10.1097/PAS.00000000000 00530

49. Weinreb JC, Barentsz JO, Choyke PL, Cornud F, Haider MA, Macura KJ, et al. PI-RADS prostate imaging-reporting and data system: 2015, version 2. Eur Urol. (2016) 69:16-40. doi: 10.1016/j.eururo.2015.08.052

50. Pathmanathan AU, As NJ van, Kerkmeijer LGW, Christodouleas J, Lawton CAF, Vesprini D, et al. Magnetic resonance imaging-guided adaptive radiation therapy: a "Game Changer" for prostate treatment? Int J Rad Oncol Biol Phys. (2018) 100:361-33. doi: 10.1016/j.ijrobp.2017.10.020

51. Moradi M, Salcudean SE, Chang SD, Jones EC, Buchan N, Casey RG, et al. Multiparametric MRI maps for detection and grading of dominant prostate tumors. J Magn Reson Imaging (2012) 35:1403-13. doi: 10.1002/jmri. 23540

52. Chaddad A, Desrosiers C, Niazi T. Deep radiomic analysis of MRI related to Alzheimer's Disease. IEEE Access. (2018) 6:58213-21. doi: 10.1109/ACCESS.2018.2871977

Conflict of Interest Statement: The authors declare that the research was conducted in the absence of any commercial or financial relationships that could be construed as a potential conflict of interest.

Copyright (C) 2018 Chaddad, Niazi, Probst, Bladou, Anidjar and Bahoric. This is an open-access article distributed under the terms of the Creative Commons Attribution License (CC BY). The use, distribution or reproduction in other forums is permitted, provided the original author(s) and the copyright owner(s) are credited and that the original publication in this journal is cited, in accordance with accepted academic practice. No use, distribution or reproduction is permitted which does not comply with these terms. 